\title{
GINGER: An array of ring lasers for testing fundamental physics
}

\author{
A. Ortolan* \\ INFN - National Laboratories of Legnaro, \\ Viale dell'Università 2, I-35020, Legnaro, Padova, Italy \\ *E-mail: ortolan@lnl.infn.it \\ J. Belfi, F. Bosi, A. Di Virgilio \\ INFN - Sez. di Pisa, Largo Pontecorvo, 2 I-56127, Pisa, Italy \\ N. Beverini, G. Carelli, E. Maccioni, R. Santagata, A. Simonelli \\ Department of Physics, University of Pisa, \\ and INFN - Sez. di Pisa, Largo Pontecorvo, 2 I-56127, Pisa, Italy
}

A. Beghi, D. Cuccato, A. Donazzan, G. Naletto

Department of Information Engineering (DEI), University of Padova, via Gradenigo 6B, I-35131, Padova, Italy, and INFN - Sez di Padova, Via Marzolo, 8 I-35131, Padova, Italy

\author{
M. G. Pelizzo \\ National Research Council - Institute for Photonics and Nanotechnology \\ CNR-IFN Padova Unit, Via Trasea 7, I-35131 Padova, Italy, \\ and INFN - Sez di Padova, Via Marzolo 8, I-35131, Padova, Italy
}

Ring laser (RL) gyroscopes are, at present, the most precise sensors of absolute angular velocity. In the near future, their application is foreseen to provide ground based tests of General Relativity. We have recently proposed a tri-axial array of RLs that can reach the sensitivity, accuracy, and long term stability required to measure the inertial frame dragging induced by the rotating Earth, as predicted by General Relativity. The effect, also known Lense-Thirring effect, amounts for the Earth to 1 part in $10^{9}$ of its rotation rate, thus requiring an unprecedented sensitivity and accuracy of experimental apparatus. An array of at least 3 RLs would allow us to measure not only the rotation rate, i.e. the angular velocity modulus, but also the angular velocity vector. In this way, having at disposal the time series of the daily estimate of Earth rotation vector from the International Earth Rotation and Reference System Service, it would be possible to isolate the Geodetic and Lense-Thirring contributions. Our proposal GINGER (Gyroscopes IN GEneral Relativity) is intended to push the present knowledge of RL physics and technology to achieve an accuracy in the estimation of the Earth rotation rate of 1 part in $10^{9}$. In the experimental apparatus we have to account for systematic errors resulting from non linear dynamics of the active laser medium, and changes of the optical cavity geometry. The redundancy of the array, e.g. the addition of a ring almost parallel to the Earth rotation axis, should allow for the reduction of such errors at the level of the geometry control. In this contribution we describe the intermediate prototypes GP2 and GEMS (GINGER External Metrology System) devoted to control the geometrical fluctuations of a RL cavity and the 3D geometry of the RL array (dihedral angles among RLs), respectively.

Keywords: General relativity; experimental gravity; ring lasers. 


\section{Introduction}

Ring Lasers (RLs) have demonstrated to be the most sensitive devices for measuring angular velocity with respect to the local inertial frame ${ }^{1,2}$. A RL usually consists of a resonant optical cavity (triangular or square) in which two laser modes, counterpropagating over the same path, are excited. By the principle of Sagnac effect, the frequencies of the two laser beams shift in response to an angular rotation $\boldsymbol{\Omega}$ of the cavity. The rotational information is then detected by measuring the frequency difference between the two counterpropagating beams (Sagnac frequency), which is given by ${ }^{1}$

$$
f_{s}=\frac{4 a}{\lambda p} \boldsymbol{\Omega} \cdot \mathbf{n}+f_{n r},
$$

where $a$ is the area enclosed by the optical path, $p$ is the optical path length (or "cavity perimeter"), $\lambda$ is the light wavelength, $\mathbf{n}$ is the unit vector normal to the instrument plane, and $f_{n r}$ represents the non-rotational contributions to the Sagnac frequency arising from non linear laser dynamics and backscattering. According to Ref. 7, the sensitivity limit of a RL for a rotation measurement arises from the irreducible quantum fluctuations of photon number (shot noise) in the interferogram. Then the variance lower bound for rotational noise is given by $\sigma_{\Omega}^{2}=[(c p) /(4 a Q)]^{2}(h \nu) /\left(P_{\text {out }} T\right)$, where $Q$ is the quality factor of the ring cavity, $\nu=c / \lambda$ the frequency of light, $P_{\text {out }}$ the detected optical power, and $T$ the integration time. This noise bound has been reached, after years of continuous improvements, by the Gross-Ring "G", a square RL with $p=16 \mathrm{~m}$, located at the Geodetic Observatory in Wettzell (Bavaria) ${ }^{2}$. "G" shows a rotational sensitivity of $\sigma_{\Omega} \simeq 0.25 \mathrm{prad} / \mathrm{s}$ with an integration time $T=10^{4} \mathrm{~s}$, which means that its resolving capability in measuring the Earth rotation rate is 3 part in $10^{9}$. Due to such technological advancements, RL appear today as a very interesting apparatus to probe the structure of space-time of GR at the laboratory scale, i.e. to measure general relativistic precessions as the de Sitter and Lense-Thirring effects. According to the theory of GR, these effects arise from the metric deformation caused by the rotating mass of the Earth which drags the local inertial frame. Einstein's equations, solved in the weak field approximation, predict a $1 \mathrm{ppb}$ correction to the Earth rotation rate calculated with respect to the local reference frame and the "fixed stars" reference frame.

The GINGER (Gyroscopes IN GEneral Relativity) project aims at measuring, for the first time in an Earth-based laboratory, such tiny effects by means of an array of large RLs. In fact, we have shown in Ref. 3 that a tri-axial system of RLs, as in GINGER, will provide an accurate estimation of the laboratory frame angular velocity vector. The comparison of the modulus of this vector with the corresponding one estimated by IERS (International Earth Rotation and Reference System Service) ${ }^{6}$, with respect to the fixed stars frame, can put in evidence the Geodetic and Lense-Thirring terms. In fact, IERS measures the Earth rotation rate with a very high accuracy of $\simeq 3 \times 10^{-15} \mathrm{rad} / \mathrm{s}$, and similar accuracy is required for GINGER. 
We note that a measurement in a terrestrial lab would give local information complementary to the space measurements based on the tracking of satellites in "free falling" orbits. Moreover, these measurements have confirmed the frame dragging effect, but they have not yet reached the full resolution that would put GR and other fundamental theories to a test ${ }^{4}$. In order to provide tests of fundamental physics (GR, Horava-Lifshitz or Chern-Simons theories of gravity, etc.), GINGER would have to improve in sensitivity and in the long-term stability with respect to " $G$ " of a factor of $\sim 5 \div 10$. In addition to this, GINGER is also committed to measuring angular velocities with very high accuracy, since the frame dragging effect appears like a small bias on top of the rate of rotation of the Earth.

\section{Relativistic Sagnac effect}

The term gravitomagnetism designates the effects and phenomena predicted by GR which occur when the source of the gravitational field is rotating. Actually, rotational effects are due also to rotating reference frames (i.e. non inertial observers), and the kinematic part of the Sagnac effect is a typical example. If the source of gravity, as for the rotating Earth, can be approximated as a steadily and freely rotating object, the line element for an observer on Earth is given by

$$
d s^{2}=\left(c^{2}+2 \mathbf{A} \cdot \mathbf{x}\right) d t^{2}-2(\boldsymbol{\Omega} \wedge \mathbf{x}) \cdot d \mathbf{x} d t-d \mathbf{x} \cdot d \mathbf{x}+O\left(|d \mathbf{x}|^{2}\right)
$$

where $t$ is the observer proper time, $\mathbf{x}$ the local Fermi coordinates, $\mathbf{A}$ the observer acceleration, and $\Omega$ the observer rotation with respect the local inertial frame whose velocity is momentarily the same as that of the accelerating observer ${ }^{5}$. Light path must satisfy the null geodetic equation $d s=0$. In the non relativistic limit, the proper-time difference $\Delta t=t_{+}-t_{-}$between the clock wise $\left(t_{+}\right)$and counter clock wise $\left(t_{-}\right)$propagation times of light along a close path $\mathcal{S}$, depends only on the time-space mixed components $(2 \Omega \wedge \mathbf{x})$ of the metric, as it is the only term that changes sign with the direction of motion, i.e.

$$
\Delta t=\frac{2}{c^{2}} \oint_{\mathcal{S}} 2(\boldsymbol{\Omega} \wedge \mathbf{x}) \cdot d \mathbf{x}=\frac{4}{c^{2}} \iint_{a} \nabla \cdot(\boldsymbol{\Omega} \wedge \mathbf{x}) d^{2} x=\frac{4 a}{c^{2}} \boldsymbol{\Omega} \cdot \mathbf{n},
$$

where the line integral is calculated on $\mathcal{S}$ with tangent vector $d \mathbf{x}$, and $a$ is the enclosed area. As RL converts time difference into frequency difference, the Sagnac frequency follows from Eq. (3) and the condition of resonance in the optical cavity $t_{+}=N T_{+}$and $t_{-}=N T_{-}$, where $T_{+}$and $T_{-}$are the periods of the two counterpropagating waves and $N \in \mathbb{N}$. The resonance condition implies $f_{s} \equiv 1 / T_{+}-1 / T_{-}=$ $c^{2} \Delta \tau /(\lambda p)=(4 a) /(\lambda p) \boldsymbol{\Omega} \cdot \mathbf{n}$.

Under the same assumptions as before, we may work out the metric elements by solving Einstein equations, and the final result reads ${ }^{3}$

$$
f_{s}=\frac{4 a}{\lambda p}\left(\boldsymbol{\Omega}_{\oplus}+\boldsymbol{\Omega}_{G}+\boldsymbol{\Omega}_{B}\right) \cdot \mathbf{n},
$$


where the effective angular velocity vector $\Omega$ sensed by a RL is the sum of 3 contributions: i) the kinematic Earth rotation vector as measured by IERS or Sagnac term $\boldsymbol{\Omega}_{\oplus} \equiv \Omega \mathbf{u}_{\oplus}$, ii) the pure mass contribution or Geodetic term $\boldsymbol{\Omega}_{G} \equiv$ $-2 G M /\left(c^{2} R\right) \Omega_{\oplus} \sin \theta \mathbf{u}_{\theta}$, and iii) the Earth angular momentum contribution or Lense-Thirring term $\boldsymbol{\Omega}_{B}=G /\left(c^{2} R^{3}\right)\left[\mathbf{J}_{\oplus}-3\left(\mathbf{J}_{\oplus} \cdot \mathbf{u}_{r}\right) \mathbf{u}_{r}\right]$; here $\mathbf{R} \equiv R \mathbf{u}_{r}$ is the position of the laboratory with respect to the center of the Earth, $\mathbf{J}_{\oplus} \simeq(2 / 5) M R^{2} \boldsymbol{\Omega}_{\oplus}$ is the angular momentum of the Earth, the unit vector $\mathbf{u}_{r}$ is along the radial direction, and $\mathbf{u}_{\theta}$ is along the local meridian in the sense of increasing colatitudes. Using Earth mass, rotation rate and radius, we have that Geodetic and Lense-Thirring terms are both of order $\sim 10^{-9}$ with respect to the Sagnac term.

\section{GINGER project}

To reach the GINGER goals, we need to improve the sensitivity and long term stability already obtained by "G" along the following lines ${ }^{3}$ :

(1) Decreasing the shot noise level. This can be obtained enlarging the RL side $L$. Note that, with equivalent mirrors quality, the sensitivity increases as $\sim L^{5}$.

(2) Increasing the long term stability by monitoring and controlling the laser operative parameters and the environment. A deep underground site seems necessary to reduce surface noise due to meteorology and seismic motion.

(3) Improving the scale factor stability and accuracy of the RLs. This implies a strict active control of the ring geometry and of its orientation with respect the Earth rotation axis. A viable solution is to implement an effective control system for the geometry stabilization of the RL optical cavity, and to use a tri-axial array of RLs. The proposed 3-dimensional geometry foresees a set of six mirrors in an octahedral configuration ${ }^{3}$.

(4) Estimating the nonlinear laser dynamics. To achieve the required accuracy, an estimate of non reciprocal effects resulting in a null-shift term in $f_{n r}$ must be implemented.

Here we present some experimental activities we are carrying out in order to address the issues of line (3), i.e. the geometry stabilization of a single RL cavity (GP2), and the control of relative orientation of different RLs in the array (GEMS).

\subsection{The GP2 RL gyroscope}

As the scale factor $k_{S}=4 a /(\lambda p)$ must be known and kept constant at the level of one part in $10^{10}$, the geometry of a RL cavity must be controlled at the same level. The GP2 prototype is conceived to test the control strategies for the RL cavity geometry, with the aim of gaining a sub-nanometer stabilization of the scale factor geometry $a / p$, going beyond the attainable level by means of passive methods. It exhibits an innovative design, in which the mirrors that constitute the square optical cavity are equipped with a piezo nano-positioning system, in order to actively control their 
relative positions. The relevant observables to constraint the geometry are the perimeter of the ring cavity and the lengths of the two diagonal cavities, defined by the mirrors on opposite corners.

The use of the diagonal lengths is pioneering in the RL technology. We have demonstrated that, when the lengths of the two diagonals are locked to the same value, the perturbations to the mirror positions affect only quadratically the ring laser perimeter and scale factor ${ }^{9}$. In this case, the regular square geometry corresponds to a saddle-point of the perimeter, and so the scale factor fluctuations are minimized. To allow the implementation of this new control strategy, the vacuum chamber of GP2 encloses also the optical paths of an external laser beam between the opposite mirrors of the cavity.

To stabilize the absolute lengths of the two diagonal cavities respect to a reference laser, an original interferometric technique has been worked out. It consists in locking the resonance frequencies $\left(f_{n}\right)$ of the two cavities to the same laser carrier frequency and, at the same time, in determining an integer multiple of the two free spectral ranges $(F S R)$. The error signals needed to implement, for each diagonal, a two frequency measurement $\left(f_{n}\right.$ and $\left.F S R\right)$ in closed loop, are recovered by phasemodulating the interrogating laser source with a combination of three independent frequencies, through a single electro-optic modulator.

The stabilization scheme has been tested in a table top experiment on two FabryPerot resonators composed by two couples of spherical mirrors. These resonators was mounted on an optical bench to simulate the cavities along the diagonals of a RL gyroscope. With this setup, we were able to demonstrate the capability of stabilizing the two lengths at the level of 1 part in $10^{11}$, and setting their difference equal to zero within the precision of $500 \mathrm{~nm}$, with residual fluctuations only limited by the laser frequency noise ${ }^{8}$. Recently, this metrology technique has been partially implemented in GP2. Preliminary results demonstrate the compatibility of the scheme with the RL operation, resulting in a sub-nanometer diagonal lengths stabilization with a signal integration time of few hundred seconds ${ }^{10}$.

\subsection{The Ginger External Metrology System (GEMS)}

As previous experience on rigid RL has already demonstrated, RLs with a passive stabilization of the cavity geometry do not allow to achieve the extremely high accuracy required for GR tests, even in the most performing condition obtained with the "G" RL. To overcome this problem, with GINGER we plan to use an active control system, capable to compensate possible sub-nanometer variation in the system geometry for all the long time of the measurements. To this end, we are considering the realization of GEMS, that is a novel metrological approach to measure and control the ring size and orientation with the required accuracy. GEMS will be integrated with GINGER RLs but will be independent of their dynamics. The basic concept of GEMS relies on a network of portable heterodyne interferometers. Each interferometer can measure the relative distance between two fiducials (refer- 
ence targets rigidly connected with the RL mirrors) with a nominal accuracy better than $100 \mathrm{pm}$. The interferometer is fed by a split frequency modulated infrared laser beam that travels either a reference path or the measurement path, and then recombines at two output photodiodes: here a suitable phase-meter measures the phase difference between the two paths and returns in real time the optical path variation along the two fiducials. By changing the laser frequency, the absolute distance measurement can be obtained as well. Once these measurements are available for each relative distance between the optical elements of the RL, the whole $3 \mathrm{D}$ geometry of the laser cavity will be determined. Moreover, depending on the number of available interferometers and the type of fiducials (either simple onedirection backreflectors, or multidirectional ones), an array of cross measurements can be realized to over-constraint the "rigidity" of the RL array. Then, thanks to a detailed mathematical model of the geometry of the RL and a multivariable control scheme based on the use of second order information on the optimization function, it will be possible to command suitable nano-positioning actuators to properly move the mirrors and actively control all the relevant dimensions of the cavities. In such a way, it will be possible to maintain a fixed geometry for GINGER within the required accuracy, independently of the environmental disturbances.

\section{Conclusions}

We have taken some experimental steps towards the implementation of the threedimensional array of large RLs GINGER. The first objective of GINGER is to measure general relativistic effects due to the rotation of the Earth. In order to investigate some key issues related to the extreme sensitivity and accuracy required by the GR measurements, we have set up the GP2 RL gyroscope to develop the control of the cavity geometry, and the GEMS prototype to develop an external metrological system that will be integrated on GINGER.

\section{References}

1. G. E. Stedman, Rep. Prog. Phys. 60, 615 (1997).

2. K. U. Schreiber and J-P. R. Wells, Rev. Sci. Instrum. 84, 041101 (2013).

3. F. Bosi, et al., Phys. Rev. D 84, 122002 (2011).

4. I. Ciufolini, et al., Class. Quantum Grav. 30, 235009 (2013).

5. C. W. Misner, K. S. Thorne and J. A. Wheeler, Gravitation (Freeman, S. Francisco, 1977).

6. http://www.iers.org/

7. K. U. Schreiber, J-P. R. Wells and G. E. Stedman, Gen. Rel. Grav. 40, 935 (2011).

8. J. Belfi, et al., Class. Quantum Grav. 31, 225003 (2014).

9. R. Santagata, et al., Class. Quantum Grav. 32, 055013 (2015).

10. R. Santagata, Sub-nanometer length metrology for ultra-stable ring laser gyroscopes (Unpublished Ph. D. Thesis), University of Siena, Italy (2015). 